\title{
EFECTO DEL EJERCICIO TERAPÉUTICO EN LA CALIDAD DE VIDA DE PACIENTES CON CÁNCER DE PULMÓN: REVISIÓN SISTEMÁTICA DE ENSAYOS CLÍNICOS ALEATORIZADOS
}

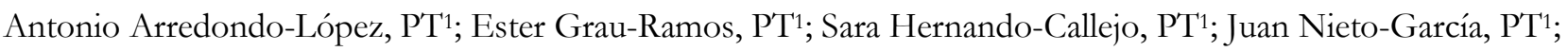 \\ Alfonso Gil-Martínez, PhD ${ }^{1-3}$. \\ 1. Departamento de Fisioterapia. Centro Superior de Estudios Universitarios La Salle. Universidad Autónoma de Madrid. Madrid. España. \\ 2. CranioSPain Research Group. Centro Superior de Estudios Universitarios La Salle. Universidad Autónoma de Madrid. Madrid. España.
}

3. Departamento de Fisioterapia. Hospital Universitario La Paz. IdiPAZ. Madrid. España.

\begin{abstract}
Correspondencia:
Alfonso Gil-Martínez, PT, PhD. Facultad de Ciencias de la Salud. CSEU La Salle. Universidad Autónoma of Madrid. Calle La Salle, $\mathrm{n}^{\circ} 10,28023$ Madrid, España Teléfono: (+34) 917401980 Fax: (+34) 913571730 E-Mail: alfonso.gil@idipaz.es

\section{Conflicto de Intereses:}

Los autores del presente manuscrito declaran no presentar ningún conflicto de interés y además el presente estudio no ha sido presentado en ningún evento científico.
\end{abstract}

\section{Financiación}

Los autores declaran no haber recibido financiación/compensación para el desarrollo de esta investigación.

DOI: https://doi.org/10.37382/jomts.v1i1.7

\section{Recepción del Manuscrito: \\ 15-·Noviembre-2018}

\section{Aceptación del Manuscrito:}

2- Enero-2019

Licensed under: CC BY-NC-SA 4.0

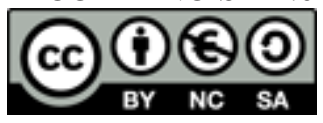

\section{RESUMEN}

Objetivo: Valorar la influencia del ejercicio terapéutico (ET) sobre la calidad de vida de pacientes con cáncer $(\mathrm{Ca})$ de pulmón. Asimismo, por su relación con la calidad de vida, se ha analizado la influencia que el ejercicio tiene sobre la tolerancia al esfuerzo y la fuerza..

Métodos: Se realizó una búsqueda de ensayos clínicos aleatorizados (ECA) en la base de datos electrónica de MEDLINE (Pubmed). Los criterios de inclusión fueron: ECA realizados en pacientes con cáncer de pulmón, bien tras intervención quirúrgica (IQ), durante o tras el tratamiento con quimioterapia, donde la intervención fuera ejercicio terapéutico, publicados en cualquier idioma. Dos revisores independientes realizaron el análisis de la calidad metodológica mediante la escala PEDro, y un tercer revisor intervino en los casos de desacuerdo.

Resultados: Se seleccionaron 8 ECA, de los cuales 4 presentaron una calidad metodológica buena, por encima de 6 , en la escala PEDro. Se ha encontrado evidencia fuerte de la influencia del ET en la mejora de la calidad de vida de los pacientes con Ca de pulmón, así como en el aumento de la tolerancia al ejercicio.

Conclusión: El ET es un tratamiento que presenta beneficios en la calidad de vida de los pacientes con Ca de pulmón. Estos beneficios no son superiores al tratamiento con fisioterapia activa convencional, terapia manual, fisioterapia respiratoria o relajación progresiva.

Palabras Clave: Ejercicio Terapéutico; Neoplasia; Calidad de vida; Tolerancia al ejercicio; Revisión sistemática. 


\section{INTRODUCCIÓN}

El cáncer (CA) es una enfermedad compleja donde concurren una serie de procesos progresivos hasta la transformación y mutación definitiva de la célula normal hacia la célula tumoral. Dicha célula mutada pierde la función habitual que realizan las células normales de un determinado órgano y termina subdividiéndose de forma progresiva, acelerada y sin límite al haber perdido su capacidad de apoptosis o muerte celular programada (Wang et al., 2015). La incidencia de CA sigue creciendo debido al aumento y envejecimiento de la población, así como un incremento de la prevalencia de los factores de riesgo como el tabaquismo, el sobrepeso, la inactividad física, y el cambio de los patrones reproductivos asociados con la urbanización y el desarrollo económico (Torre et al., 2015). La falta de síntomas clínicos en la enfermedad en estadio temprano conduce con frecuencia al diagnóstico (Dx) en una etapa avanzada, con una tasa de supervivencia de 5 años (Reddy et al., 2011).

El CA de pulmón es la principal causa de muerte tanto en hombres como mujeres superando en el caso de las mujeres de países desarrollados al CA de mama (De Flora et al., 1996; Torre et al., 2015). El proceso de CA de pulmón es similar al de otros tipos de CA. La célula normal que se transforma en la célula tumoral se encuentra en el epitelio que reviste todo el árbol respiratorio desde la traquea hasta el bronquiolo terminal más fino y las células que se encuentran en los alveolos pulmonares (Wang et al., 2015). Como hemos mencionado anteriormente el Dx suele ser tardío, en fase ya metastásica, esta es la razón principal relacionada con el fracaso en el tto y en la supervivencia de los pacientes (Pastorino, 2010). El cribado anual que se realiza con radiografía de tórax y análisis citológico del esputo, no es suficiente para reducir la mortalidad, debido a la agresividad y heterogeneidad (Oken et al., 2011).

A nivel mundial el CA de pulmón es el más frecuente (Adiguzel y Kulah, 2014), con una tasa de mortalidad superior al CA de mama, próstata y colon (Brad G.Smith, M.S, CCC-SLP, Jan S. Lewis, 2014).
En 2008 se estima que aparecieron aproximadamente 1,6 millones de casos nuevos y 1,4 millones de muertes por CA de pulmón en todo el mundo (Ferlay et al., 2010; Jemal et al., 2011). En España se diagnostican unos 20.000 casos anuales, lo que representa el $18,4 \%$ de los tumores entre los hombres (18.000 casos) y el 3,2\% entre las mujeres (2.000 casos), la mayoría se diagnostican entre los 55 y los 75 años, con un máximo entre los 65 y los 70 años (Asociación Española Contra el Cáncer, 2017). Sin embargo, el CA de pulmón sigue siendo la principal causa de muerte en España por enfermedad tumoral en los hombres y el tercero más común en las mujeres, 20.755 hombres y 3.452 mujeres en 2010 (Sánchez de Cos Escuín et al., 2013).

La calidad de vida de los pacientes que padecen CA de pulmón se ve muy influenciada, entendiendo calidad de vida como un concepto multidimensional que engloba el bienestar físico (relacionado con la actividad funcional), social (relacionado con las funciones y relaciones sociales), psicológico (relacionados con emociones) y espiritual (engloba el significado de la enfermedad, la esperanza, transcendencia) (Loh y Musa, 2015).

El Ejercicio Terapéutico (ET) consiste en la prescripción de un programa de Actividad Física que involucra al paciente en la tarea voluntaria de realizar una contracción muscular y/o movimiento corporal con el objetivo de aliviar los síntomas, mejorar la función y mantener o frenar el deterioro de la salud (Thompson et al., 2013). Aunque existe evidencia creciente que parece indicar que la terapia con ejercicio es útil para disminuir el nivel de ansiedad y depresión (Chen et al., 2015), y algunos autores han demostrado su eficacia en la reducción los síntomas físicos, aumentando la tolerancia al ejercicio, mejorando la calidad de vida, y reduciendo la duración de la estancia y las complicaciones postoperatorias en pacientes con CA de pulmón (Bade et al., 2015; Quist et al., 2015) es necesario seguir investigando la efectividad del ET sobre los pacientes con CA, con el fin de obtener mayor evidencia y consensuar una guía de referencia para el empleo adecuado del ET en estos pacientes. 
En base a lo expuesto, el objetivo de esta revisión es revisar el efecto del ET sobre la calidad de vida de pacientes con CA de pulmón.

\section{MÉTODOS}

\section{Criterios de inclusión de los estudios}

Los criterios de selección utilizados en esta revisión se basan en aspectos metodológicos y clínicos como el tipo de estudio, la población de estudios, las intervenciones y medidas para los resultados.

\section{Tipo de estudios}

Se seleccionaron ensayos clínicos aleatorizados (ECAs) que presentasen comparaciones con un grupo control $u$ otras intervenciones debidamente protocolizadas. Se incluyen estudios publicados en todos los idiomas y sin restricción por fecha de la publicación. Ver Figura 1.

\section{Pacientes}

Los pacientes de los ensayos seleccionados debían ser mayores de 18 años, diagnosticados de cáncer de pulmón.

\section{Intervención terapéutica}

Fueron incluidos ECAs en los que las intervenciones principales se basaran en ET combinado o no a otros tratamientos de fisioterapia.

\section{Medidas de los resultados}

Las medidas para comprobar los resultados y efectos del tratamiento debían valorar al menos la variable de calidad de vida.

\section{Estrategia de búsqueda}

Se realizó una búsqueda de artículos científicos utilizando la base de datos MEDLINE a través de su motor de búsqueda PubMed durante el mes de abril de 2015 (hasta 30/04/15). La estrategia de búsqueda generada por el motor de búsqueda de la base de datos PubMed fue la siguiente: (("exercise"[MeSH Terms] OR "exercise"[All Fields]) AND ("lung neoplasms"[MeSH Terms] OR ("lung"[All Fields] AND "neoplasms"[All Fields]) OR "lung neoplasms"[All Fields] OR ("lung"[All Fields] AND "cancer"[All Fields]) OR "lung cancer"[All Fields]) AND ("quality of life"[MeSH Terms] OR ("quality"[All Fields] AND "life"[All Fields]) OR "quality of life"[All Fields])) AND Randomized Controlled Trial[ptyp]).

La fase de búsqueda la realizaron cuatro revisores de forma conjunta.

Figura 1. Diagrama de flujo de los artículos seleccionados en la Revisión Sistemática.

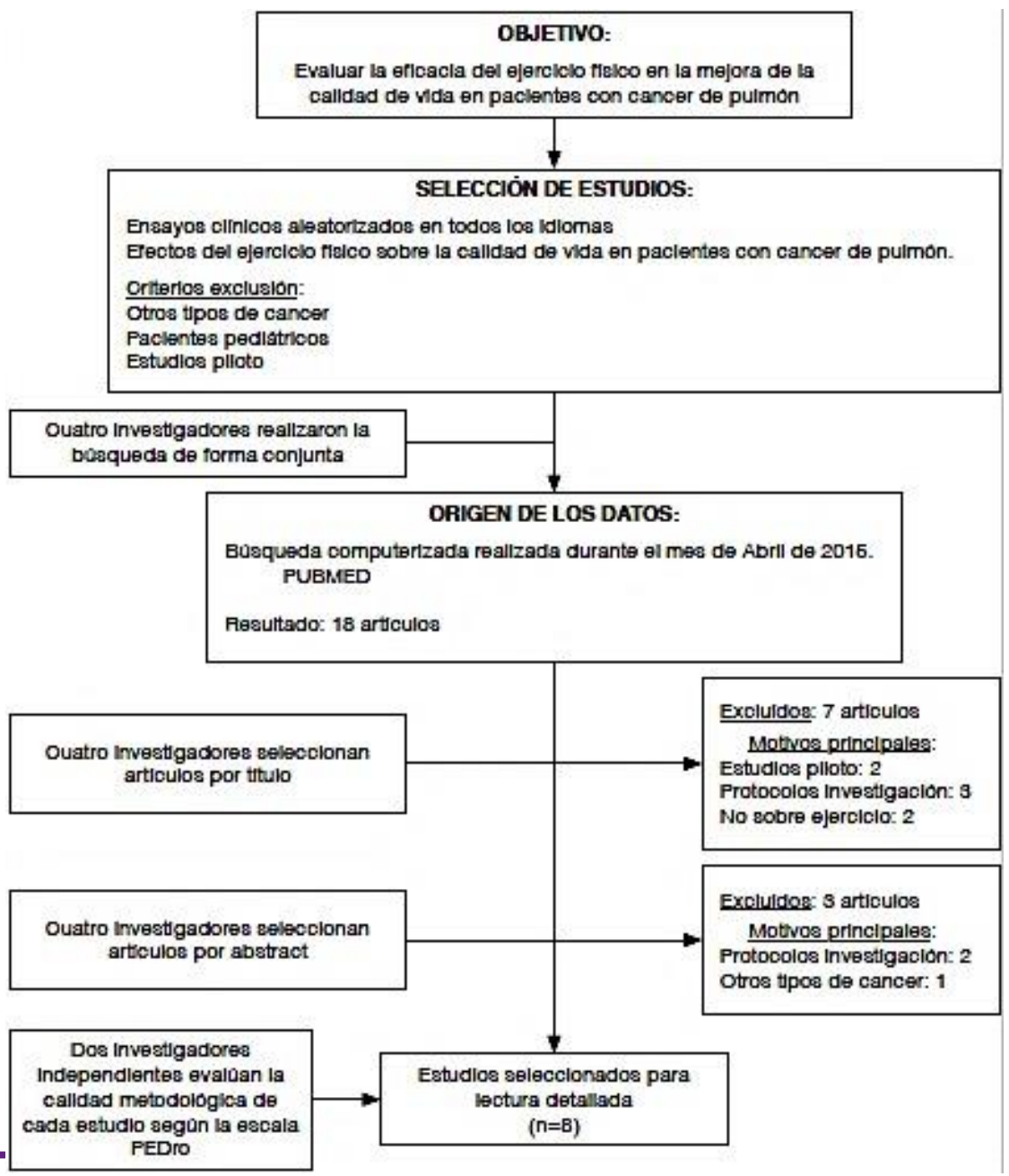




\section{Criterios de selección y extracción de datos}

El primer análisis de información la realizaron cuatro investigadores que evaluaron la idoneidad de los ECAs en relación con el objetivo de investigación. Este primer análisis se efectuó basándose en la información del título. En la segunda fase de análisis, se comprobó la utilidad y el cumplimiento de los criterios de inclusión de los ECAs mediante la revisión del resumen. Por último, se llevó a cabo una tercera fase, en la que se revisó a texto completo los ensayos clínicos restantes.

\section{Valoración de la calidad de los estudios}

La valoración de calidad metodológica de los estudios, se realizó mediante la escala PEDro traducida y validada al español, la cual consta de 11 ítems (Morton, 2009).

Para mantener la consistencia con la escala Delphi (Verhagen et al., 1998), la escala PEDro descarta por defecto el primer ítem dejando el número de ítems a evaluar en 10. Los criterios metodológicos se califican de esta forma: se cumple (1 punto) y no se cumple o se desconoce ( 0 puntos). La máxima puntuación es de 10 puntos, con un baremo de 0 a 10 . Se consideran estudios de calidad aceptable cuando cumplen seis o más criterios (Maher et al., 2003).

Dos revisores independientes analizaron la calidad metodológica de todos los artículos seleccionados. Los desacuerdos entre revisores se resolvieron por consenso mediante la inclusión de un tercer revisor.

\section{Análisis cualitativo}

El análisis cualitativo utilizado en esta revisión se basó en la clasificación de los resultados según los niveles de evidencia científica (Furlan et al., 2009). La evidencia fue categorizada en cinco niveles, dependiendo de la calidad metodológica de los estudios:

- Evidencia fuerte: representa resultados de múltiples ECAs con buena calidad metodológica.

- Evidencia moderada: representa resultados de múltiples ECAs con baja calidad metodológica, ensayos clínicos controlados o un ECA de alta calidad.

- Evidencia limitada: representa resultados de un ECA o un ensayo clínico controlado de baja calidad.

- Evidencia contradictoria: representa resultados contradictorios de ECAs o ensayos clínicos controlados.

- No hay evidencia: no existen ECAs ni ensayos clínicos controlados.

Un requisito indispensable para describir los resultados y conclusiones según los niveles de evidencia científica es la homogeneidad clínica y metodológica en los estudios. Los resultados descritos en el formato que incluyen los niveles de evidencia se pueden observar en los apartados de resultados y conclusión.

\section{RESULTADOS}

Para la presente revisión, se seleccionaron 8 ECAs de los cuales 4 de ellos presentaban una buena calidad metodológica, mientras que los 4 restantes no alcanzaban el baremo de 6 puntos según la escala PEDro (Tabla 1).

\section{Características de la intervención}

De los ECAs analizados y con puntuación de 6 o más en la escala PEDro, dos han tenido seguimientos entorno a las 4 semanas (Arbane et al., 2014; Morano et al., 2014), otro 3 semanas (Dimeo et al., 2004), siendo el ECA de Brocki et al. el único que realiza seguimiento a medio-largo plazo, realizando evaluaciones a 4 y 12 meses (Brocki et al., 2014). En total, 238 pacientes pertenecieron al grupo de intervención, mientras que 234 lo hicieron al grupo control. La edad media de la muestra fue de 69,6 años (mín.-max.; 32-82), de los que el 57\% fueron hombres.

En todos ellos, se evalúa la calidad de vida, cuatro de ellos lo hacen utilizando la escala EORTC QLQ C-30 1C-13, tres emplean la escala SF-36 (Stigt et al., 2013; Brocki et al., 2014; Morano et al., 2014) y el otro utiliza ambas escalas (Furlan et al., 2009). 
Tabla 1. Puntuación de ensayos clínicos aleatorizados en la escala PEDro.

\begin{tabular}{|c|c|c|c|c|c|c|c|c|c|c|c|c|}
\hline ESCALA PEDro & 1 & 2 & 3 & 4 & 5 & 6 & 7 & 8 & 9 & 10 & 11 & TOTAL \\
\hline G Arbane et al. , 2014 & 1 & 1 & 1 & 1 & 0 & 0 & 0 & 0 & 1 & 1 & 1 & 7 \\
\hline Brocki et al. ,2014 & 1 & 1 & 1 & 1 & 0 & 0 & 1 & 0 & 1 & 1 & 1 & 8 \\
\hline D. Thomas et al. ,2004 & 1 & 1 & 1 & 1 & 0 & 0 & 0 & 1 & 1 & 0 & 1 & 7 \\
\hline Morano et al. ,2014 & 1 & 1 & 0 & 1 & 0 & 0 & 0 & 1 & 1 & 1 & 1 & 7 \\
\hline G Arbane et al. ,2011 & 1 & 1 & 0 & 1 & 0 & 0 & 0 & 0 & 0 & 1 & 1 & 5 \\
\hline Henke et al. , 2014 & 1 & 1 & 0 & 1 & 0 & 0 & 0 & 0 & 0 & 1 & 1 & 5 \\
\hline Hwang, Yu et al. ,2012 & 1 & 0 & 0 & 0 & 0 & 0 & 1 & 1 & 0 & 1 & 1 & 5 \\
\hline Stigt et al. ,2013 & 1 & 1 & 0 & 0 & 0 & 0 & 0 & 1 & 0 & 1 & 1 & 6 \\
\hline
\end{tabular}

Todos ellos tienen en común la inclusión de diferentes programas de ET en la intervención asignada a los grupos de experimentales. En uno de los ECAs, el ET se añadió a la intervención de fisioterapia convencional del grupo control (Furlan et al., 2009). En otros dos ECAs (Dimeo et al., 2004; Morano et al., 2014) se comparó la intervención con ET frente a la intervención con fisioterapia respiratoria convencional y programa de relajación respectivamente. En otro de los ensayos la comparación es entre un programa de ET supervisado frente a uno no supervisado (Brocki et al., 2014).

En dos ECAs la intervención con ET incluyó la supervisión del mismo durante todo el período del estudio (Dimeo et al., 2004; Morano et al., 2014), en otro de ellos la supervisión se realizó durante las cuatro primeras semanas y se continuó durante los ocho meses siguientes con monitorización mediante visitas semanales (Brocki et al., 2014). Por último, en el estudio de Arbane et al. se supervisó a los pacientes mientras estaban hospitalizados pero no lo estuvieron tras el alta (Arbane et al., 2014).

En cuanto a los programas de ET prescritos, podemos indicar que la mayoría de los ECAs incluyeron el trabajo aeróbico combinado con el trabajo de fuerza muscular (Arbane et al., 2014; Brocki et al., 2014; Morano et al., 2014) mientras el restante sólo incluyó trabajo aeróbico (Dimeo et al., 2004). El trabajo aeróbico en la mayoría de los casos se realizaba en bicicleta estática (Dimeo et al., 2004; Arbane et al., 2014) o en tapiz rodante (Morano et al., 2014). Cuando la intervención se continuaba en domicilio se sustituye la bicicleta por la marcha. En lo que respecta a la frecuencia de las sesiones se proponía trabajo aeróbico la mayoría de días de la semana, en cambio el trabajo de fuerza se pautó con una frecuencia menor. Es interesante destacar el estudio de Brocki et al. ya que la frecuencia era de un día a la semana para ambos tipos de trabajo mientras era supervisado, y luego aumentaba la frecuencia cuando era domiciliario y por tanto no supervisado (Brocki et al., 2014). 
La duración de las sesiones de la intervención, varía entre los 30 minutos del ensayo de Dimeo et al. (Dimeo et al., 2004) y los 60 minutos del ensayo de Brocki et al. (Brocki et al., 2014). En la Tabla 2 se representan los principales datos y resultados de cada estudio.

\section{DISCUSIÓN}

El análisis realizado en esta revisión, basado en los resultados y conclusiones de los estudios seleccionados, describe efectos positivos del ET combinado con otras terapias o aplicado como única terapia, para la mejora de la calidad de vida y la tolerancia al ejercicio de los pacientes con cáncer que han sido intervenidos quirúrgicamente o que han recibido o reciben tratamiento quimioterápico en la actualidad. Es importante destacar que el ET no ha demostrado ser estadísticamente mejor que otras terapias como los protocolos de fisioterapia convencional, terapia de relajación progresiva, fisioterapia respiratoria, o incluso el ET no supervisado.

De los ocho estudios seleccionados cuatro presentaron una aceptable calidad metodológica y obtuvieron 6 o más puntos en la escala PEDro (Arbane et al., 2014; Morano et al., 2014).

\section{Calidad de vida}

Si bien no se han encontrado diferencias entre grupos en la variable calidad de vida en la mayoría de los estudios incluidos en esta Revisión, debemos indicar que existe una tendencia a favor del grupo de intervención (ET supervisado) frente al grupo control (ET no supervisado) en la evaluación intermedia a los cuatro meses (Brocki et al., 2014). Estos datos podrían indicar una recuperación más rápida de los niveles de calidad de vida, llegando a valores similares a los de la población general, sin embargo en la medición a los doce meses los valores entre grupos se equiparan (Brocki et al., 2014).

En relación al estudio de Morano et al. es necesario indicar la tendencia favorable encontrada en el componente físico de la calidad de vida (SF-36) en favor del ET frente a la fisioterapia respiratoria (Morano et al., 2014).

En el ensayo de Arbane et al. (2014), se encontraron mejoras significativas de la calidad de vida en los pacientes del grupo intervención (terapia convencional combinado con ET) frente al grupo control (terapia convencional) al realizar un análisis secundario en el subgrupo de pacientes con cáncer de pulmón y además diagnosticados de EPOC (Arbane et al., 2014). Los resultados fueron positivos en el cuestionario SF-36, sin embargo esta mejora no se encontró para los mismos pacientes en el cuestionario EORTC QLQ C-30 1C-13 (Arbane et al., 2014). Es posible que, en este tipo de pacientes con EPOC, la inclusión de ET adicional a la terapia convencional sea de mayor importancia para su recuperación y podría ser una línea de investigación prometedora para futuros ECAs.

Dos artículos de calidad metodológica deficiente sí indican mejoras significativas en la calidad de vida respecto al grupo de intervención. En uno de ellos, los sujetos que recibían fisioterapia convencional combinada con ET mejoraron en la variables de funcionamiento físico del SF-36 frente a los grupos que recibía fisioterapia convencional (Henke et al., 2014). El otro ECA con resultados muy similares, comparaba el ET supervisado frente a no supervisado (Stigt et al., 2013). Serán necesarios ECAs de alta calidad metodológica que confirmen estos hallazgos.

El hecho de que no se hayan encontrado diferencias en la calidad de vida en diferentes grupos de estudio, podría deberse a que los pacientes de los grupos de control, mayoritariamente, estaban recibiendo tratamientos convencionales basados en estrategias activas. De esta manera, se podría pensar que todas aquellas estrategias activas de tratamiento, como es el ejercicio activo o ejercicios respiratorios, podrían mostrarse beneficiosas en la calidad de vida de los pacientes con CA de pulmón. 
Tabla 2. Características epidemiológicas de los estudios, resultados y conclusiones de cada uno de ellos.

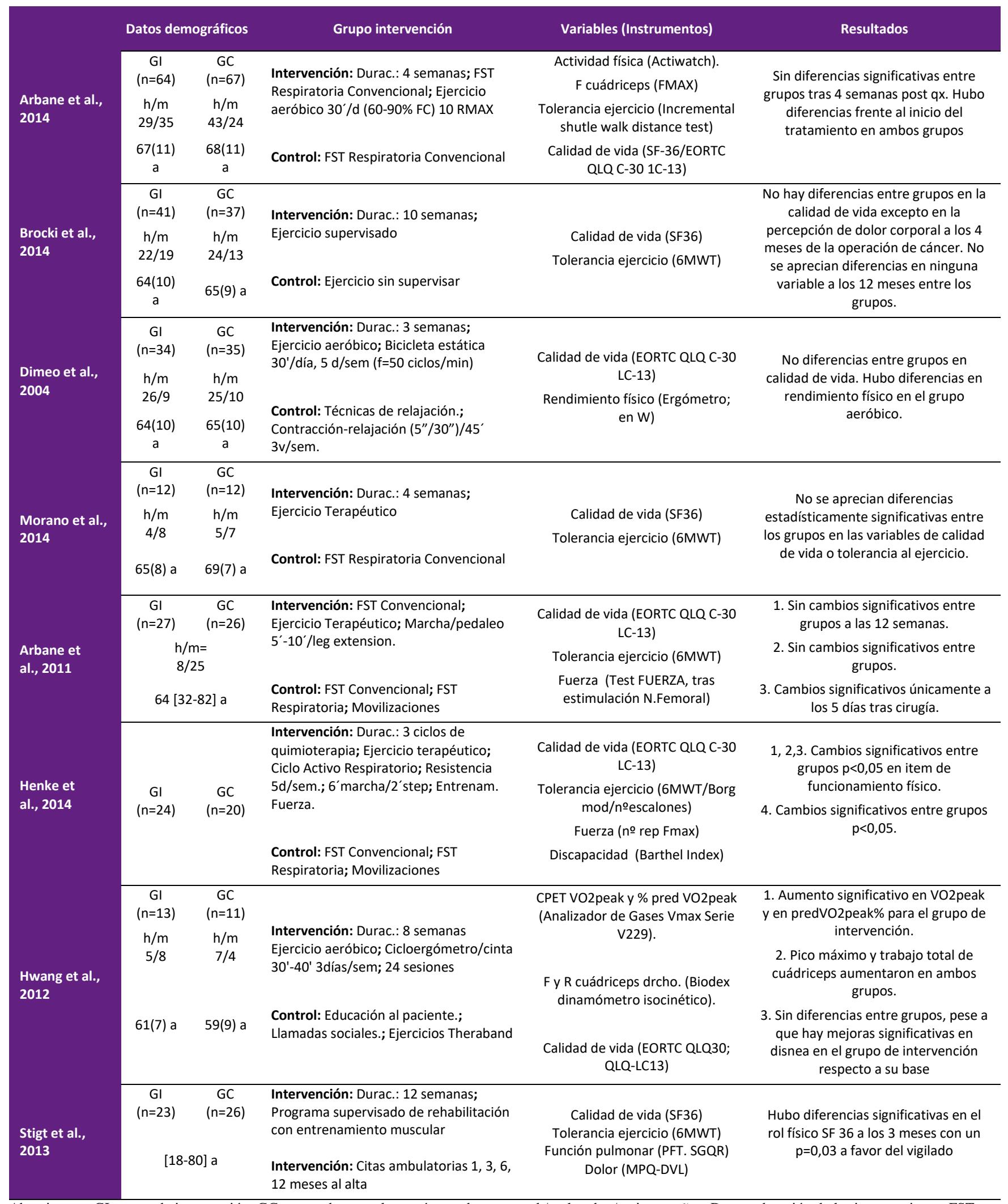

Abreviaturas: GI: grupo de intervención; GC: grupo de control; n= número de muestra; h/m: hombre/mujer; a: años; Durac.: duración de las intervenciones; FST: fisioterapia; FC: frecuencia cardíaca; RMÁX: repetición máxima; F: fuerza; CdV: calidad de vida; Qx: cirugía; FMAX: fuerza máxima; SF36: cuestionario corto de calidad de vida relacionado con la salud; EORTC QLQ C-30 LC-13: Cuestionario de calidad de vida en cáncer de pulmón de la Organización Europea para la Investigación y Tratamiento del Cáncer ; 6MWT: test de la marcha de 6 minutos; '/día: minutos por día; d/sem: días por semana; f: frecuencia; min: minutos; ": segundos; v/sem: veces por semana; W: vatios; N. Femoral: nervio femoral; Borg mod: escala de esfuerzo Borg modificada; $\mathrm{N}^{\circ}$ escalones: número peldaños ejecutados en 2 minutos; Rep: repeticiones; CPET VO2peak: pico de flujo en test de ejercicio cardiopulmonar; F y R: fuerza y resistencia; Drcho: derecho; Vmáx: volumen máximo; PFT. SGQR: cuestionario San Jorge de test función pulmonar; MPQ-DVL: versión holandesa del cuestionario de dolor McGill 


\section{Tolerancia al ejercicio}

En lo que a la variable tolerancia al ejercicio se refiere, el análisis basado en los resultados y conclusiones de los estudios seleccionados describe efectos positivos del ET, tanto combinado con otras terapias como aplicado de forma independiente. En tres de los estudios analizados (Arbane et al., 2014; Brocki et al., 2014; Morano et al., 2014), no se aprecian diferencias significativas entre el grupo de intervención de ET frente a los grupos de control a los que aplicaban fisioterapia convencional (fisioterapia respiratoria y terapia manual), ET no supervisado o fisioterapia respiratoria exclusivamente. En uno de los estudios se refieren mejoras significativas del grupo de intervención con ET frente al de relajación progresiva, si bien la variable medida en este estudio es la de rendimiento físico (Dimeo et al., 2004).

Es posible que la no existencia de diferencias significativas entre la terapia con ET se pueda deber por un lado al corto período de tiempo de los estudios, 4 semanas en dos de ellos (Arbane et al., 2014; Morano et al., 2014) y 3 semanas en otro (Dimeo et al., 2004), lo que dificulta la progresión en el ejercicio y la posible falta de adherencia cuando no se está en el entorno hospitalario. Además las pruebas realizadas como el 6MWT son submáximas y no permiten poner en evidencia diferencias significativas entre los pacientes. Seguramente las dosis de ejercicio propuestas en cuanto a frecuencia, intensidad y duración no consiguen generar adaptaciones en tan cortos períodos de tiempo.

\section{Comentarios adicionales}

El único estudio que ha evaluado los resultados a corto y largo plazo (Brocki et al., 2014) da una tendencia mejor en el grupo de ET coincidiendo con el período en el que efectivamente éste estaba siendo supervisado, y eso a pesar de que en su muestra inicial, el $43 \%$ de los pacientes del grupo de ejercicio no supervisado ya declaraba hacer ejercicio antes del estudio, lo que podría indicar que estaban adheridos a la terapia.
En otro de los estudios en el que se compara ET frente a técnicas de relajación (Dimeo et al., 2004), parece lógico entender que no existan diferencias en la calidad de vida ya que cada terapia incide en parámetros diferentes de los cuestionarios de calidad de vida. Este resultado sugiere la importancia que tiene para estos pacientes un abordaje multidisciplinar que tenga en cuenta los factores físicos, emocionales y psicológicos.

Serán necesarios estudios que tengan en cuenta los parámetros FITT (Frecuencia, Intensidad, Tiempo y Tipo) del ejercicio para estos pacientes así como la adherencia al mismo, la supervisión, seguimiento, monitorización, los tiempos de adaptación y progresión, así como test de evaluación que pongan en evidencia las diferencias.

Revisiones sistemáticas previas a la realizada han obtenido resultados similares en cuánto a la variable calidad de vida, no encontrando diferencias significativas, salvo para algún parámetro concreto, y encontrando diferencias más importantes en cuánto a la capacidad o tolerancia al ejercicio se refiere. Todas estas revisiones indican la necesidad de estudios de mayor calidad metodológica (Granger et al., 2011; Braam et al., 2016). Asimismo otra revisión sistemática indica la no existencia de un programa definido adecuado para este tipo de pacientes (Crandall et al., 2014).

La revisión realizada parece indicar que el ET debe ser incluido en los protocolos de tratamiento de los pacientes de cáncer de pulmón, adecuándolo de forma individualizada a cada paciente y realizando un seguimiento adecuado, esta idea coincide con las conclusiones con otros investigadores (Bourke et al., 2013). La educación será fundamental para conseguir la adherencia requerida para que la terapia activa sea exitosa.

\section{Implicaciones clínicas}

Según la evidencia obtenida en esta revisión sistemática, consideramos necesario la inclusión ejercicio y estrategias activas de tratamiento, en combinación con otras intervenciones fisioterápicas o 
de forma independiente, para mejorar la calidad de vida y tolerancia al ejercicio de los pacientes con cáncer de pulmón.

\section{Líneas futuras y recomendaciones}

A pesar de que el ejercicio ha estado históricamente contraindicado en pacientes con CA, parece que en la actualidad existe suficiente literatura que apoya su uso para mejorar la calidad de vida de estos pacientes. Para un adecuado prescripción de ejercicio en estos pacientes, se recomienda desarrollar programas de ejercicio que establezcan las dosis, frecuencia, intensidad, tipo y tiempo, que unifique este tipo de tratamiento $\mathrm{y}$ facilite su comparación con otras terapias convencionales. Parece que es en la prescripción de la dosis adecuada a cada paciente así como en la adherencia a la terapia, donde puede estar la clave del éxito. El abandono de las terapias podría suponer una disminución importante de eficacia de la propia intervención (Andersen et al., 2011). En esta línea, Temel et al. indican que una estrategia para mejorar la adherencia al ejercicio podría ser realizarlo de forma colectiva (Temel et al., 2009). En la revisión sistemática Cochrane de Bourke et al. encuentran que los elementos que más favorecen la adherencia al ejercicio son el establecimiento de objetivos, la automonitorización y que los pacientes reproduzcan hábitos adquiridos en la etapa supervisada (Bourke et al., 2013). Algunos sistemas basado en gamificación podrían también ser de utilidad para estos pacientes.

La terapia con ET requiere de tiempos mayores para la consecución de resultados que otras terapias por lo que los seguimientos a los pacientes deberán ampliarse para dar tiempo a generar adaptaciones y cambios conductuales relacionados con la actividad física. En este sentido coincidimos con las recomendaciones de la revisión Cochrane de Mishra et al. (Mishra et al., 2012), donde indican que, para la obtención de resultados más significativos, sería recomendable mantener el seguimiento de los pacientes a medio y largo plazo (a partir de 12 meses).

\section{Limitaciones}

Existen una serie de limitaciones en la presente revisión. En primer lugar, la búsqueda se ha realizado sólo en la base de datos electrónica de MEDLINE (PubMed), que, aunque es la base de datos con más potencial para ciencias de la salud con más de 28 millones de referencias y artículos, no asegura que hayamos encontrado toda la literatura disponible en este momento. Los resultados de la presente revisión, deben tomarse con precaución, ya que tan sólo 4 de los 8 estudios incluidos, tienen una aceptable calidad metodológica. A pesar de que en la mayoría de estudios donde hay intervenciones de fisioterapia, es difícil hacer enmascaramientos, se recomienda que próximos estudios realicen una asignación aleatoria de los sujetos a los grupos y que se realice adecuada, al menos, ente el cegamiento a los evaluadores.

La variabilidad en las propuestas de ET posible y la combinación con otras terapias hace difícil concluir si los efectos se deben a una u otra terapia de las utilizadas. Incluso podría dificultar la realización de un meta-análisis debido la heterogeneidad de las variables utilizadas. El seguimiento realizado a los pacientes en la mayoría de los ECAs evaluados es de corta duración y esto puede ser una limitación teniendo en cuenta que el uso de ET pretende mantener los efectos sobre diferentes variables a medio y largo plazo.

\section{CONCLUSIÓN}

Existe evidencia fuerte de los efectos positivos que el ET ejercicio en general tiene en la mejora de calidad de vida y el aumento de la tolerancia al ejercicio en pacientes con $\mathrm{CA}$ de pulmón, bien combinada con otras terapias o como terapia única. No existe evidencia de que la intervención con ET sea superior a otras formas de ejercicio. 


\section{FRASES DESTACADAS}

$>$ El ejercicio terapéutico mejora la calidad de vida y aumenta de la tolerancia al ejercicio en pacientes con cáncer de pulmón.

$>$ El ejercicio terapéutico se puede utilizar como terapia única o como terapia combinada con otros tratamientos en el cáncer de pulmón.

$>$ No existe evidencia de que la intervención con ejercicio terapéutico sea superior a otras formas de ejercicio en pacientes con cáncer de pulmón.

\section{REFERENCIAS}

Adiguzel Y, Kulah H. Breath sensors for lung cancer diagnosis. Vol. 65C, Biosensors \& bioelectronics. 2014. p. 121-38.

Andersen AH, Vinther A, Poulsen L-L, Mellemgaard A. Do patients with lung cancer benefit from physical exercise? Acta Oncol. 2011;50(2):307-13.

Arbane G, Douiri A, Hart N, Hopkinson NS, Singh S, Speed C, et al. Effect of postoperative physical training on activity after curative surgery for non-small cell lung cancer: a multicentre randomised controlled trial. Physiotherapy. 2014;100(2):100-7.

Arbane G, Tropman D, Jackson D, Garrod R. Evaluation of an early exercise intervention after thoracotomy for non-small cell lung cancer (NSCLC), effects on quality of life, muscle strength and exercise tolerance: randomised controlled trial. Lung Cancer. 2011;71(2):229-34.

Asociación Española Contra el Cáncer. ¿Qué es cáncer? AECC. 2017. p. 1.

Bade BC, Thomas DD, Scott JB, Silvestri GA. «Increasing Physical Activity and Exercise in Lung Cancer: Reviewing Safety, Benefits, and Application». J Thorac Oncol. 2015;10(6):861-71.

Bourke L, Homer KE, Thaha MA, Steed L, Rosario DJ, Robb $\mathrm{KA}$, et al. Interventions for promoting habitual exercise in people living with and beyond cancer. Cochrane database Syst Rev. 2013;24(9):CD010192.

Braam KI, van der Torre P, Takken T, Veening MA, van Dulmen-den Broeder E, Kaspers GJL. Physical exercise training interventions for children and young adults during and after treatment for childhood cancer. Cochrane database Syst Rev. 2016;31(3):CD008796.

Brad G.Smith, M.S, CCC-SLP, Jan S. Lewis PD. The Role of Lymphedema Management in Head and Neck Cancer. Curr Opin Otolaryngol Head Neck Surg. NIH Public Access. 2014;18(3):153-8.
Brocki BC, Andreasen J, Nielsen LR, Nekrasas V, GorstRasmussen A, Westerdahl E. Short and long-term effects of supervised versus unsupervised exercise training on healthrelated quality of life and functional outcomes following lung cancer surgery - a randomized controlled trial. Lung Cancer. 2014;83(1):102-8.

Chen H-M, Tsai C-M, Wu Y-C, Lin K-C, Lin C-C. Randomised controlled trial on the effectiveness of home-based walking exercise on anxiety, depression and cancer-related symptoms in patients with lung cancer. $\mathrm{Br} \mathrm{J}$ Cancer. 2015;112(3):438-45.

Crandall K, Maguire R, Campbell A, Kearney N. Exercise intervention for patients surgically treated for Non-Small Cell Lung Cancer (NSCLC): a systematic review. Surg Oncol. 2014;23(1):17-30.

Dimeo FC, Thomas F, Raabe-Menssen C, Propper F, Mathias M. Effect of aerobic exercise and relaxation training on fatigue and physical performance of cancer patients after surgery. A randomised controlled trial. Support Care Cancer. 2004;12(11):774-9.

Ferlay J, Shin H-R, Bray F, Forman D, Mathers C, Parkin DM. Estimates of worldwide burden of cancer in 2008 GLOBOCAN 2008. Int J Cancer. 2010;127(12):2893-917.

De Flora S, Izzotti A, Randerath K, Randerath E, Bartsch H, Nair $\mathrm{J}$, et al. DNA adducts and chronic degenerative disease. Pathogenetic relevance and implications in preventive medicine. Mutat Res. 1996;366(3):197-238.

Furlan AD, Pennick V, Bombardier C, van Tulder M. 2009 updated method guidelines for systematic reviews in the Cochrane Back Review Group. Spine (Phila Pa 1976). 2009;34(18):1929-41.

Granger CL, McDonald CF, Berney S, Chao C, Denehy L. Exercise intervention to improve exercise capacity and health related quality of life for patients with Non-small cell lung cancer: a systematic review. Lung Cancer. 2011;72(2):139-53.

Henke CC, Cabri J, Fricke L, Pankow W, Kandilakis G, Feyer $\mathrm{PC}$, et al. Strength and endurance training in the treatment of lung cancer patients in stages IIIA/IIIB/IV. Support Care Cancer. 2014;22(1):95-101

Jemal A, Bray F, Center MM, Ferlay J, Ward E, Forman D. Global cancer statistics. CA Cancer J Clin. 2011;61(2):6990.

Loh SY, Musa AN. Methods to improve rehabilitation of patients following breast cancer surgery: a review of systematic reviews. Breast cancer (Dove Med Press. 2015;11(7):8198.

Maher CG, Sherrington C, Herbert RD, Moseley AM, Elkins M Reliability of the PEDro scale for rating quality of randomized controlled trials. Phys Ther. 2003;83(8):71321.

Mishra SI, Scherer RW, Geigle PM, Berlanstein DR, Topaloglu $\mathrm{O}$, Gotay CC, et al. Exercise interventions on health-related quality of life for cancer survivors. Cochrane database Syst Rev. 2012;15(8):CD007566. 
Morano MTAP, Mesquita R, Da Silva GPF, Araujo AS, Pinto JMDS, Neto AG, et al. Comparison of the effects of pulmonary rehabilitation with chest physical therapy on the levels of fibrinogen and albumin in patients with lung cancer awaiting lung resection: a randomized clinical trial. BMC Pulm Med. 2014;28(14):121.

Morton NA De. The PEDro scale is a valid measure of the methodological quality of clinical trials : a demographic study. Aust J Physiother. Elsevier; 2009;55(2):129-33.

Oken MM, Hocking WG, Kvale PA, Andriole GL, Buys SS, Church TR, et al. Screening by chest radiograph and lung cancer mortality: the Prostate, Lung, Colorectal, and Ovarian (PLCO) randomized trial. JAMA. 2011;306(17):1865-73.

Pastorino U. Lung cancer screening. $\mathrm{Br} \mathrm{J}$ Cancer. Nature Publishing Group; 2010;102(12):1681-6.

Quist M, Adamsen L, Rorth M, Laursen JH, Christensen KB, Langer SW. The Impact of a Multidimensional Exercise Intervention on Physical and Functional Capacity, Anxiety, and Depression in Patients With Advanced-Stage Lung Cancer Undergoing Chemotherapy. Integr Cancer Ther. 2015;14(4):341-9.

Reddy C, Chilla D, Boltax J. Lung cancer screening: a review of available data and current guidelines. Hosp Pract (1995). 2011;39(4):107-12.
Sánchez de Cos Escuín J, Serra Mitjans M, Hernández Hernández J, Hernández Rodríguez H. The Spanish Society of Pulmonology and Thoracic Surgery Lung Cancer Cooperative Group-II Registry. A Descriptive Study. Arch Bronconeumol. SEPAR; 2013;49(11):462-7.

Stigt JA, Uil SM, van Riesen SJH, Simons FJNA, Denekamp M, Shahin GM, et al. A randomized controlled trial of postthoracotomy pulmonary rehabilitation in patients with resectable lung cancer. J Thorac Oncol. 2013;8(2):214-21.

Temel JS, Greer JA, Goldberg S, Vogel PD, Sullivan M, Pirl WF, et al. A structured exercise program for patients with advanced non-small cell lung cancer. J Thorac Oncol. 2009;4(5):595-601.

Thompson P, Arena R, Riebe D, Pescatello L, Medicine AC of S. ACSM's new preparticipation health screening recommendations from ACSM's guidelines for exercise testing and prescription, ninth edition. Curr Sport Med Rep. 2013;12(4):215-7.

Torre LA, Bray F, Siegel RL, Ferlay J, Lortet-Tieulent J, Jemal A. Global cancer statistics, 2012. CA Cancer J Clin. 2015;65(2):87-108.

Verhagen AP, de Vet HC, de Bie RA, Kessels AG, Boers M, Bouter LM, et al. The Delphi list: a criteria list for quality assessment of randomized clinical trials for conducting systematic reviews developed by Delphi consensus. J Clin Epidemiol. 1998;51(12):1235-41.

Wang L, Yu J, Xu J, Zheng C, Li X, Du J. The analysis of microRNA-34 family expression in human cancer studies comparing cancer tissues with corresponding pericarcinous tissues. Gene. 2015;554(1):1-8. 